\title{
The Correlation between Synthetic Oxytocin Given during Labor and Women's Personality Traits According to the SSP (Swedish University Scales of Personality) Survey: A Pilot Study
}

\author{
Eva Wiberg-Itzel ${ }^{1}$, Kerstin Uvnäs-Moberg² \\ ${ }^{1}$ Department of Clinical Science and Education, Karolinska Institute, Women's Clinic, Sodersjukhuset, Sweden \\ ${ }^{2}$ Department of Animal Environment and Health, Swedish University of Agricultural Sciences, Skara, Sweden \\ Email: Wiberg-itzel@ki.se
}

How to cite this paper: Wiberg-Itzel, E. and Uvnäs-Moberg, K. (2021) The Correlation between Synthetic Oxytocin Given during Labor and Women's Personality Traits According to the SSP (Swedish University Scales of Personality) Survey: A Pilot Study. Open Journal of Obstetrics and Gynecolo$g y, 11,701-712$.

https://doi.org/10.4236/ojog.2021.116065

Received: May 5, 2021

Accepted: June 12, 2021

Published: June 15, 2021

Copyright $\odot 2021$ by author(s) and Scientific Research Publishing Inc. This work is licensed under the Creative Commons Attribution International License (CC BY 4.0).

http://creativecommons.org/licenses/by/4.0/

\begin{abstract}
Background: In modern obstetric care, oxytocin is one of the most frequently used drugs, and the possible mental impact this drug has on women is very little studied. The objective of this study is to investigate whether women augmented with oxytocin during labor will rate their personality profile differently after childbirth than non-stimulated women. Methods: Prospective cohort study was performed at Women's Clinic, Soder hospital, Stockholm.76 women received the SSP (Swedish University Scales of Personality) questionnaire to fill in during their stay in the post-maternity ward after labor. Information about the use of oxytocin was retrieved from the women's medical records. Primary outcome: Differences in the SSP scores in the group augmented with synthetic oxytocin during labor compared with the non-augmented group. Results: Women with and without oxytocin estimates on the SSP subscale form differed regarding personality traits described as "lack of assertiveness" ( $p=0.04$ ), which means "lack of ability to speak up and to be self-assertive in social situations". The result also showed that women that had a long time of augmentation with oxytocin ( $>5 \mathrm{~h}$ ) scored higher for "social desirability" ( $p=0.004)$, which was defined as being "socially adapted," "friendly," and "helpful". A difference in "psychological anxiety" ( $p=0.04)$ and "social desirability" ( $p=0.004)$ was found among women who had oxytocin in a dose of at least $200 \mathrm{ImU} / \mathrm{h}$ for $\geq 1$ hour. This group also had a lower rate of "mental anxiety" than those who received lower oxytocin doses. Conclusion: Synthetic oxytocin given during labor may affect the woman mentally. The total time and volume of given oxytocin seem to be essential factors when discussing augmentation's maternal psychological response. We con-
\end{abstract}


clude that prolonged and extended use of synthetic oxytocin during labor should be avoided if possible.

\section{Keywords}

Anxiety, Augmentation, Oxytocin, Personality Traits

\section{Introduction}

Oxytocin is a hormone produced in the brain's hypothalamic part (the supraoptic and the paraventricular nuclei). It is excreted into the bloodstream from nerves projecting to the pituitary gland. In modern obstetric care, oxytocin is one of the most frequently used drugs, primarily in prolonged, dystocic deliveries [1]. Synthetic oxytocin mimics the body's endogenous oxytocin system; it stimulates uterine contractions and can prevent postpartum bleeding.

Oxytocin is secreted into the circulation and areas in the Central Nervous System (CNS) via oxytocin-containing nerves originating from the paraventricular nucleus. By influencing the brain's regulatory regions, oxytocin induces many behavioral and physiological processes [2] [3] [4]. Oxytocin stimulates interactive social behaviors and exerts pain-relieving, sedative, and anxiolytic effects and therapeutic effects. It is released by sensory nerves' stimulation during labor, suckling, sexual stimulation, and touch, and probably also by different forms of positive and friendly social interaction [2] [3] [4] [5]. Oxytocin released during childbirth in women decreases pain and may increase the newly delivered woman's emotional well-being [2]. It is well known that oxytocin released in connection with birth plays an essential role in the bonding and interaction between a mother and her newborn infant after labor. It reduces maternal anxiety and stress levels and stimulates social skills and social interaction.

How synthetic oxytocin is given during birth influences the activity in the endogenous oxytocin system in the brain is not known. Since oxytocin is a hydrophilic and polar neuropeptide, it does not cross the blood-brain barrier and, consequently, does not penetrate the brain's blood circulation [5]. On the other hand, synthetic oxytocin increases uterine contractions and may increase the release of endogenous oxytocin within the brain because of enhanced activity in the Fergusson reflex [6] [7].

In modern obstetric care, oxytocin is one of the most frequently used drugs, and the possible mental impact on the woman this drug has is very little studied. This ignorance makes this work essential both from the aspect of women and the obstetric society.

This project's objective was to investigate whether women who have given birth and received oxytocin during labor rate their personality profile (SSP; Swedish University Scales of Personality) differently from women who did not receive oxytocin during labor. 


\section{Material and Method}

\subsection{Study Population}

A prospective multicentre trial-the "Dysfunctional Labour Study"-was performed in delivery wards in Sweden, Switzerland, France, and Tanzania between 2010 and 2014 [8]. The regional ethics committee at the Karolinska Institute, Stockholm, Sweden (diary 2010/199-31/1) approved the Dysfunctional Labour Study. Nulliparous women in active labor were invited to take part in the study. The study's inclusion criteria were first-time mothers with a singleton pregnancy, gestational age between $37-42$ weeks, and no maternal/fetal chronic and pregnancy-related conditions. Spontaneous onset of labor, regular contractions, and cervical dilation of at least $3 \mathrm{~cm}$ were required before the women were invited to participate in the study. Oxytocin was then given to all women, where labor was considered dystocic according to clinical guidelines. At Soder Hospital in Stockholm, 100 of the women included consecutively in the Dysfunctional Labour Study also received the SSP questionnaire to fill in after labor, during their stay in the post-maternity ward [9].

The SSP survey was collected only at Soder Hospital for a limited period during the study; therefore, just a little part of the study population was included. The SSP subscale form was completed 1 - 3 days postpartum before the woman left the hospital.

Obstetric background and labor data from all deliveries included in the study were reported in a shared internet database completed by a local research midwife. The database was password protected and encrypted. All personal data were encoded so that individuals could not be identified in the analysis. The result of the SSP subscale form was also included in the database.

\subsection{SSP Personality Inventory (Swedish University Scales of Personality)}

The SSP is a personality inventory validated and used in other similar contexts to describe personality traits [2] [3] [4] It contains 91 questions with four possible response options. The test response is then divided into 13 different subscales, scored according to a specially developed model. The 13 personality traits that the questions highlight is: somatic trait anxiety, psychic trait anxiety, stress susceptibility, lack of assertiveness, impulsiveness, adventure-seeking, detachment, social desirability, and embitterment, trait irritability, mistrust, verbal trait aggression, and physical trait aggression. The scores of the 13 subscales were then compared with an available standard scale for a normative non-preg- nant group of women.

The 100 women included in the study were divided into two groups, one of which received oxytocin during labor and did not. The oxytocin group was further examined for how long-time oxytocin was administered and the maximum dose obtained during augmentation. The questionnaire's results were then analysed according to whether oxytocin was given, how long it was given, and in 
what amount. Maternal and neonatal background and labor data were collected from the women's medical files after labor.

In Sweden, the "low dose" of oxytocin is the standard dose. The Swedish National Board recommends oxytocin preparation with $1 \mathrm{ml}$ oxytocin $(8,3 \mathrm{mi}$ $\mathrm{krog} / \mathrm{ml}=5 \mathrm{IU} / \mathrm{ml})$, mixed in $500 \mathrm{ml}$ isotone saline solution $(9 \mathrm{mg} / \mathrm{ml})$. The infusion is started with $20 \mathrm{ml} / \mathrm{h}(100 \mathrm{mIU} / \mathrm{h})$, and the rate is increased by $20 \mathrm{ml}$ every 20 minutes to a maximum dose of $180 \mathrm{ml} / \mathrm{h}$ (900 mIU/h). An adjusted dosage according to individual response and the lowest effective treatment is recommended.

\subsection{Statistics}

Histograms were initially used to assess data distribution. As some of the data were not normally distributed, and there were no differences in clinical significance between the mean and median values, continuous variables were presented as medians with min and max values. Other maternal and fetal characteristics are presented as frequencies. Maternal pregnancy outcome characteristics and the questionnaire's answers were compared between the two groups using the chi-square test and Fisher's exact test in small groups. For non-normally distributed values, the comparison was performed by Mann-Whitney U-test.

For the statistical analyses, the Statistica ${ }^{\circledR}$ program (13.2) was used. P-values < 0.05 were considered statistically significant. The STROBE cohort reporting guidelines are used in the primary "Dysfunctional Labour Study" and this substudy [10].

\section{Results}

100 women were included in the study from the beginning. Twenty-four of them did not fill in the questionnaire correctly and were consequently excluded in line with the correction template of SSP. The remaining 76 women constituted our material. No statistically significant difference was found between the two groups (oxytocin or not) according to maternity age, BMI, or smoking. Neither neonatal background data such as gestational age, newborn weight, neonatal length, and head circumference (Table 1 ).

$74 \%(56 / 76)$ of the women included received oxytocin at some time during labor. The median time of augmentation was 3 hours $(0.0-11.5 \mathrm{~h})$. When oxytocin was initiated, the median cervical dilation was $7 \mathrm{~cm}(2-10 \mathrm{~cm})$. The median maximum dose of oxytocin given during labor was $90 \mathrm{ml} / \mathrm{h}(450 \mathrm{mIU} / \mathrm{h})$. No differences were obtained in the rate of spontaneous vaginal delivery between the two groups (oxytocin given vs. not given, $82 \%$ vs. $90 \%, \mathrm{p}=0.7$ ), neither in terms of vacuum delivery ( $11 \%$ vs. $5 \%, \mathrm{p}=0.7)$ nor emergency cesarean section ( $7 \%$ vs. $5 \%, p=0.7$, Table 1$) .88 \%(49 / 56)$ in the oxytocin group also received an epidural (EDA) compared with $30 \%(6 / 20)$ in the non-oxytocin group ( $\mathrm{p}<0.001$, Table 1$)$. The latent phase was statistically more extended (7.0 vs. 3.3 $h, p=0.03)$ in the oxytocin group compared to the group without augmentation. 
Table 1. Maternal and fetal background and delivery data presented as median values with range (min-max). Proportions are described as percentages $(\%)(\mathrm{N}=76)$.

\begin{tabular}{|c|c|c|c|c|}
\hline Maternal & $\begin{array}{l}\text { Oxytocin } \\
\text { No }(n=20)\end{array}$ & $\begin{array}{l}\text { Oxytocin } \\
\text { Yes }(n=56)\end{array}$ & p-value* & $\begin{array}{c}\text { Total } \\
(\mathrm{n}=76)\end{array}$ \\
\hline Maternal age (years) & $\begin{array}{c}30 \\
(20-39)\end{array}$ & $\begin{array}{c}30 \\
(21-39)\end{array}$ & 0.9 & $\begin{array}{c}30 \\
(20-39)\end{array}$ \\
\hline $\mathrm{BMI}^{* *}$ & $\begin{array}{c}22 \\
(19-27)\end{array}$ & $\begin{array}{c}23 \\
(18-29)\end{array}$ & 0.2 & $\begin{array}{c}22.6 \\
(17.5-28.5)\end{array}$ \\
\hline Smoking (\%) & $\begin{array}{c}1 \\
(5.0)\end{array}$ & $\begin{array}{c}2 \\
(3.6)\end{array}$ & 0.6 & $\begin{array}{c}3 \\
(4.0)\end{array}$ \\
\hline Education; university (yes, \%) & $13(65)$ & $32(57.1)$ & 0.5 & $\begin{array}{c}45 \\
(59.2)\end{array}$ \\
\hline Psychiatric Contact (\%) & $\begin{array}{c}5 \\
(25.0)\end{array}$ & $\begin{array}{c}11 \\
(19.6)\end{array}$ & 0.4 & $\begin{array}{c}16 \\
(21.0)\end{array}$ \\
\hline \multicolumn{5}{|l|}{ Foetal } \\
\hline Gestational age & $\begin{array}{c}40 \\
(37-41)\end{array}$ & $\begin{array}{c}40 \\
(37-42)\end{array}$ & 0.9 & $\begin{array}{c}40 \\
(37-42)\end{array}$ \\
\hline Fetal Weight $(\mathrm{g})^{*}$ & $\begin{array}{c}3600 \\
(2740-4540)\end{array}$ & $\begin{array}{c}3480 \\
(2555-4975)\end{array}$ & 0.7 & $\begin{array}{c}3527 \\
(2555-4975)\end{array}$ \\
\hline Fetal Length $(\mathrm{cm})$ & $\begin{array}{c}51 \\
(45-56)\end{array}$ & $\begin{array}{c}51 \\
(47-55)\end{array}$ & 0.8 & $\begin{array}{c}50.8 \\
(45-56)\end{array}$ \\
\hline Head circumference $(\mathrm{cm})$ & $\begin{array}{c}34.8 \\
(31-37)\end{array}$ & $\begin{array}{c}35 \\
(32-38)\end{array}$ & 0.8 & $\begin{array}{c}34.7 \\
(31-38)\end{array}$ \\
\hline Gender (boy, \%) & $\begin{array}{c}9 \\
(45.0)\end{array}$ & $\begin{array}{c}27 \\
(48.0)\end{array}$ & 0.5 & $\begin{array}{c}36 \\
(47.4)\end{array}$ \\
\hline \multicolumn{5}{|l|}{ Delivery data } \\
\hline $\begin{array}{l}\text { Labor start (\%) } \\
\text { Spontaneous } \\
\text { Induction }\end{array}$ & $\begin{array}{c}18(90) \\
2(10)\end{array}$ & $\begin{array}{c}52(93) \\
4(7)\end{array}$ & 0.7 & $\begin{array}{c}70(92) \\
6(8)\end{array}$ \\
\hline $\begin{array}{l}\text { Labor end (\%) Spontaneous Vaginal } \\
\text { Vacuum Acute caesarean section }\end{array}$ & $\begin{array}{l}18(90) \\
1(5) 1(5)\end{array}$ & $\begin{array}{c}46(82) \\
6(11) 4(7)\end{array}$ & 0.7 & $\begin{array}{c}64(84) \\
7(9) 5(7)\end{array}$ \\
\hline Latent phase (h) & $\begin{array}{c}3.3 \\
(0-41)\end{array}$ & $\begin{array}{c}7.0 \\
(0-65)\end{array}$ & $0.03^{*}$ & $\begin{array}{c}6.5 \\
(0-65)\end{array}$ \\
\hline Active phase (h) & $\begin{array}{c}8.0 \\
(0.5-17.0)\end{array}$ & $\begin{array}{c}6.5 \\
(1.5-15.0)\end{array}$ & 0.1 & $\begin{array}{c}7.8 \\
(0.5-17.0)\end{array}$ \\
\hline Fully dilated until delivery (h) & $\begin{array}{c}1.0 \\
(0-2.0)\end{array}$ & $\begin{array}{c}1.5 \\
(0.3-5.0)\end{array}$ & $<0.001^{*}$ & $\begin{array}{c}1.5 \\
(0-5)\end{array}$ \\
\hline Epidural (yes) & $6(30)$ & $49(88)$ & $<0.001^{\star}$ & $56(73)$ \\
\hline $\mathrm{aPh}$ in cord blood $\leq 7.10(\%)$ & 0 & $2(3.6)$ & 0.6 & $2(2.6)$ \\
\hline
\end{tabular}

${ }^{\star} \mathrm{P}$ values $<0.05$ are statistically significant.

No difference in the active stage of labor was found (6.5 vs. $8.0 \mathrm{~h}, \mathrm{p}=0.10)$. Oxytocin-stimulated women were fully dilated, on average, 1.5 hours vs. 1.0 hours for those who did not receive oxytocin $(\mathrm{p}<0.001)$.

Analysis of the women's estimates in the SSP subscale form, personality trait number four, relating to "lack of assertiveness," was statistically different when 
comparing the groups (oxytocin or not). "Lack of assertiveness" is defined according to the SSP's template as a perceived sense of "lack of ability to speak up and to be self-assertive in social situations" ( $\mathrm{p}=0.04$, Table 2 ).

Women who had oxytocin for a longer time ( $\geq 5 \mathrm{~h})$ scored higher on question number eight ("social desirability," $\mathrm{p}=0.04$, Table 3) compared to those who received oxytocin for a shorter time during labor. "Social desirability" is defined in the SSP format as "socially adapted," "friendly," and "helpful." The median value among women stimulated for more than 5 hours was $3.00(2.43-3.86)$ (Table 3).

$49 \%(37 / 76)$ of the women included in the study estimated a higher score of "social desirability" than the median value described, and of these, $78 \%(29 / 37)$ received oxytocin $\geq 5$ hours (Table 3 ).

To investigate the importance of the time aspect combined with the dose of oxytocin given during labor, different combinations of augmentation time and doses were tested statistically. A significant difference in the women's estimates

Table 2. Women's estimated score in SSP correlated to whether they received oxytocin during labor or not. The values are presented as median values (min-max). $\mathrm{N}=76$.

\begin{tabular}{|c|c|c|c|c|c|}
\hline $\begin{array}{c}\text { SSP scales } \\
\text { (median values) }\end{array}$ & $\begin{array}{l}\text { No oxytocin } \\
\quad(n=20)\end{array}$ & $\begin{array}{l}\text { Oxytocin } \\
(n=56)\end{array}$ & P-values* & $\begin{array}{l}\text { Median value among } \\
\text { included women }\end{array}$ & $\begin{array}{l}\text { Normative value among } \\
\text { non-pregnant women }\end{array}$ \\
\hline 1. Somatic Anxiety Trait & $\begin{array}{c}1.78 \\
(1.3-2.4)\end{array}$ & $\begin{array}{c}1.87 \\
(1.0-3.3)\end{array}$ & 0.6 & 1.85 & 1.96 \\
\hline 2. Psychological Anxiety Trait & $\begin{array}{c}1.81 \\
(1.0-2.86)\end{array}$ & $\begin{array}{c}2.02 \\
(1.0-3.71)\end{array}$ & 0.05 & 1.86 & 2.13 \\
\hline 3. Stress Susceptibility & $\begin{array}{c}1.79 \\
(1.14-3.0)\end{array}$ & $\begin{array}{c}1.98 \\
(1.0-3.86)\end{array}$ & 0.2 & 1.86 & 2.07 \\
\hline 4. Lack of Assertiveness & $\begin{array}{c}1.83 \\
(1.14-3.0)\end{array}$ & $\begin{array}{c}2.08 \\
(1.14-3.29)\end{array}$ & $0.04^{*}$ & 2.0 & 2.15 \\
\hline 5. Impulsive Ness & $\begin{array}{c}2.12 \\
(1.43-3.00)\end{array}$ & $\begin{array}{c}2.22 \\
(1.14-3.14)\end{array}$ & 0.3 & 2.29 & 2.28 \\
\hline 6. Adventure Seeking & $\begin{array}{c}2.47 \\
(1.71-3.29)\end{array}$ & $\begin{array}{c}2.40 \\
(1.29-3.57)\end{array}$ & 0.8 & 2.43 & 2.44 \\
\hline 7. Detachment & $\begin{array}{c}1.79 \\
(1.00-2.86)\end{array}$ & $\begin{array}{c}1.77 \\
(1.00-3.43)\end{array}$ & 0.9 & 1.71 & 1.95 \\
\hline 8. Social Desirability & $\begin{array}{c}2.87 \\
(2.00-3.57)\end{array}$ & $\begin{array}{c}2.92 \\
(2.00-3.86)\end{array}$ & 0.7 & 2.86 & 2.88 \\
\hline 9. Embitterment & $\begin{array}{c}1.58 \\
(1.00-2.14)\end{array}$ & $\begin{array}{c}1.61 \\
(1.14-2.71)\end{array}$ & 0.9 & 1.57 & 1.79 \\
\hline 10. Irritability Trait & $\begin{array}{c}2.61 \\
(1.43-3.57)\end{array}$ & $\begin{array}{c}2.50 \\
(1.17-3.71)\end{array}$ & 0.5 & 2.57 & 2.35 \\
\hline 11. Mistrust & $\begin{array}{c}1.75 \\
(1.0-3.71)\end{array}$ & $\begin{array}{c}1.73 \\
(1.00-3.57)\end{array}$ & 0.8 & 1.71 & 1.93 \\
\hline 12. Verbal Aggression Trait & $\begin{array}{c}2.29 \\
(1.57-3.43)\end{array}$ & $\begin{array}{c}2.07 \\
(1.00-2.29)\end{array}$ & 0.2 & 2.14 & 2.10 \\
\hline 13. Psychological Aggression Trait & $\begin{array}{c}1.65 \\
(1.00-3.29)\end{array}$ & $\begin{array}{c}1.62 \\
(1.00-2.71)\end{array}$ & 0.5 & 1.43 & 1.89 \\
\hline
\end{tabular}

${ }^{*} \mathrm{P}$ values $<0.05$ are statistically significant. 
Table 3. The estimate of personality related to oxytocin use $<$ or $>5 \mathrm{~h}$ during labor. The values are presented as median values (min-max). $\mathrm{N}=76$.

\begin{tabular}{|c|c|c|c|c|c|c|}
\hline $\begin{array}{c}\text { SSP scales } \\
\text { (median values) }\end{array}$ & $\begin{array}{l}\text { Oxytocin }<5 \mathrm{~h} \\
\quad(\mathrm{n}=41)\end{array}$ & $\begin{array}{l}\text { Oxytocin } \geq 5 \mathrm{~h} \\
\quad(\mathrm{n}=20)\end{array}$ & $\begin{array}{l}\text { No Oxytocin } \\
\quad(n=15)\end{array}$ & P-värde* & $\begin{array}{l}\text { Median value among } \\
\text { included women }\end{array}$ & $\begin{array}{l}\text { Normative value among } \\
\text { non-pregnant women }\end{array}$ \\
\hline 1. Somatic Anxiety Trait & $\begin{array}{c}1.86 \\
(1.00-3.29)\end{array}$ & $\begin{array}{c}1.84 \\
(1.00-2.86)\end{array}$ & $\begin{array}{c}1.83 \\
(1.3-2.4)\end{array}$ & 0.99 & 1.85 & 1.96 \\
\hline $\begin{array}{l}\text { 2. Psychological Anxiety } \\
\text { Trait }\end{array}$ & $\begin{array}{c}1.86 \\
(1.00-3.71)\end{array}$ & $\begin{array}{c}1.93 \\
(1.00-3.57)\end{array}$ & $\begin{array}{c}1.57 \\
(1.0-2.86)\end{array}$ & 0.6 & 1.86 & 2.13 \\
\hline 3. Stress Susceptibility & $\begin{array}{c}1.86 \\
(1.00-3.57)\end{array}$ & $\begin{array}{c}1.93 \\
(1.0-3.86)\end{array}$ & $\begin{array}{c}1.57 \\
(1.14-3.0)\end{array}$ & 0.4 & 1.86 & 2.07 \\
\hline 4. Lack of Assertiveness & $\begin{array}{c}2.00 \\
(1.14-3.29)\end{array}$ & $\begin{array}{c}2.00 \\
(1.43-3.29)\end{array}$ & $\begin{array}{c}1.86 \\
(1.14-3.0)\end{array}$ & 0.5 & 2.0 & 2.15 \\
\hline 5. Impulsive Ness & $\begin{array}{c}2.29 \\
(1.14-3.14)\end{array}$ & $\begin{array}{c}2.29 \\
(1.29-3.00)\end{array}$ & $\begin{array}{c}2.14 \\
(1.43-3.00)\end{array}$ & 0.8 & 2.29 & 2.28 \\
\hline 6. Adventure Seeking & $\begin{array}{c}2.43 \\
(1.29-3.57)\end{array}$ & $\begin{array}{c}2.43 \\
(1.33-3.29)\end{array}$ & $\begin{array}{c}2.29 \\
(1.71-3.29)\end{array}$ & 0.9 & 2.43 & 2.44 \\
\hline 7. Detachment & $\begin{array}{c}1.71 \\
(1.00-3.43)\end{array}$ & $\begin{array}{c}1.71 \\
(1.14-2.71)\end{array}$ & $\begin{array}{c}1.86 \\
(1.00-2.86)\end{array}$ & 0.4 & 1.71 & 1.95 \\
\hline 8. Social Desirability & $\begin{array}{c}2.77 \\
(2.00-3.57)\end{array}$ & $\begin{array}{c}3.00 \\
(2.43-3.86)\end{array}$ & $\begin{array}{c}2.87 \\
(2.00-3.57)\end{array}$ & $0.04^{*}$ & 2.86 & 2.88 \\
\hline 9. Embitterment & $\begin{array}{c}1.59 \\
(1.14-2.71)\end{array}$ & $\begin{array}{c}1.64 \\
(1.14-2.43)\end{array}$ & $\begin{array}{c}1.58 \\
(1.00-2.14)\end{array}$ & 0.6 & 1.57 & 1.79 \\
\hline 10. Irritability Trait & $\begin{array}{c}2.42 \\
(1.43-3.57)\end{array}$ & $\begin{array}{c}2.55 \\
(1.17-3.71)\end{array}$ & $\begin{array}{c}2.61 \\
(1.43-3.57)\end{array}$ & 0.4 & 2.57 & 2.35 \\
\hline 11. Mistrust & $\begin{array}{c}1.72 \\
(1.00-3.57)\end{array}$ & $\begin{array}{c}1.71 \\
(1.00-2.86)\end{array}$ & $\begin{array}{c}1.75 \\
(1.0-3.71)\end{array}$ & 0.95 & 1.71 & 1.93 \\
\hline $\begin{array}{l}\text { 12. Verbal } \\
\text { Aggression Trait }\end{array}$ & $\begin{array}{c}2.06 \\
(1.14-3.00)\end{array}$ & $\begin{array}{c}2.05 \\
(1.00-3.29)\end{array}$ & $\begin{array}{c}2.29 \\
(1.57-3.43)\end{array}$ & 0.9 & 2.14 & 2.10 \\
\hline $\begin{array}{l}\text { 13. Psychological } \\
\text { Aggression Trait }\end{array}$ & $\begin{array}{c}1.62 \\
(1.00-2.71)\end{array}$ & $\begin{array}{c}1.51 \\
(1.00-2.57)\end{array}$ & $\begin{array}{c}1.65 \\
(1.00-3.29)\end{array}$ & 0.4 & 1.43 & 1.89 \\
\hline
\end{tabular}

${ }^{\star} \mathrm{P}$ values $<0.05$ are statistically significant.

on the SSP form was obtained among women who had oxytocin at a dose of 40 $\mathrm{ml} / \mathrm{h}(200 \mathrm{mIU} / \mathrm{h})$ or more for at least 1 hour or more. A significant difference was noted for "psychological anxiety" (group $2, \mathrm{p}=0.04$ ) and for "social desirability" (group $8, \mathrm{p}=0.004$ ). Those who received oxytocin at a level of $40 \mathrm{ml} / \mathrm{h}$ $(200 \mathrm{IU} / \mathrm{h})$ for at least one $\mathrm{h}$ had a lower rate of "mental anxiety" than those who received lower doses of oxytocin. Similarly, those who received $40 \mathrm{ml} / \mathrm{h}(200$ IU/h) for at least one h scored higher in terms of "social desirability" compared to those who did not receive oxytocin (Table 4). No detectable difference was seen at lower doses given.

\section{Discussion}

This study shows that women who had synthetic oxytocin during labor are less affected by anxiety and scored higher in terms of "social desirability" postpartum than a normative group of women. For how long time these effects persist is still unknown. An association with the amount of oxytocin given was shown, but 
Table 4. Estimation of SSP correlated to oxytocin stimulation for at least 1 hour and at a particular dose. The values are presented as median values (min-max). $\mathrm{N}=76$.

\begin{tabular}{|c|c|c|c|c|c|c|}
\hline $\begin{array}{c}\text { SSP Scales } \\
\text { (median values) }\end{array}$ & $\begin{array}{c}\text { Oxytocin }<1 \mathrm{~h} \text { and } \\
40 \mathrm{ml}(\mathrm{n}=13)\end{array}$ & $\begin{array}{l}\text { Oxytocin } \geq 1 \mathrm{~h} \\
\text { and } 40 \mathrm{ml}(\mathrm{n}=43)\end{array}$ & $\begin{array}{l}\text { No oxytocin } \\
\quad(\mathrm{n}=20)\end{array}$ & P-value* & $\begin{array}{l}\text { Median value among } \\
\text { included women }\end{array}$ & $\begin{array}{l}\text { Normative value among } \\
\text { non-pregnant women }\end{array}$ \\
\hline 1. Somatic Anxiety Trait & $\begin{array}{c}1.80 \\
(1.29-2.71)\end{array}$ & $\begin{array}{c}1.90 \\
(1.00-3.29)\end{array}$ & $\begin{array}{c}1.86 \\
(1.30-2.40)\end{array}$ & 0.5 & 1.85 & 1.96 \\
\hline $\begin{array}{l}\text { 2. Psychological Anxiety } \\
\text { Trait }\end{array}$ & $\begin{array}{c}2.25 \\
(1.57-3.71)\end{array}$ & $\begin{array}{c}1.93 \\
(1.00-3.00)\end{array}$ & $\begin{array}{c}1.86 \\
(1.0-2.86)\end{array}$ & $0.04^{*}$ & 1.86 & 2.13 \\
\hline 3. Stress Susceptibility & $\begin{array}{c}1.90 \\
(1.29-3.57)\end{array}$ & $\begin{array}{c}2.01 \\
(1.00-3.86)\end{array}$ & $\begin{array}{c}1.57 \\
(1.14-3.0)\end{array}$ & 0.6 & 1.86 & 2.07 \\
\hline 4. Lack of Assertiveness & $\begin{array}{c}2.16 \\
(1.14-3.29)\end{array}$ & $\begin{array}{c}2.04 \\
(1.29-3.29)\end{array}$ & $\begin{array}{c}1.86 \\
(1.14-3.0)\end{array}$ & 0.4 & 2.0 & 2.15 \\
\hline 5. Impulsive Ness & $\begin{array}{c}2.21 \\
(1.29-3.14)\end{array}$ & $\begin{array}{c}2.22 \\
(1.14-3.14)\end{array}$ & $\begin{array}{c}2.29 \\
(1.43-3.00)\end{array}$ & 0.9 & 2.29 & 2.28 \\
\hline 6. Adventure Seeking & $\begin{array}{c}2.60 \\
(1.43-2.57)\end{array}$ & $\begin{array}{c}2.32 \\
(1.29-3.43)\end{array}$ & $\begin{array}{c}2.71 \\
(1.71-3.29)\end{array}$ & 0.1 & 2.43 & 2.44 \\
\hline 7. Detachment & $\begin{array}{c}1.67 \\
(1.00-2.43)\end{array}$ & $\begin{array}{c}1.81 \\
(1.42-3.43)\end{array}$ & $\begin{array}{c}1.87 \\
(1.00-2.86)\end{array}$ & 0.3 & 1.71 & 1.95 \\
\hline 8. Social Desirability & $\begin{array}{c}2.83 \\
(2.00-3.86)\end{array}$ & $\begin{array}{c}3.21 \\
(2.43-3.86)\end{array}$ & $\begin{array}{c}2.87 \\
(2.00-3.57)\end{array}$ & $0.004^{*}$ & 2.86 & 2.88 \\
\hline 9. Embitterment & $\begin{array}{c}1.57 \\
(1.29-2.71)\end{array}$ & $\begin{array}{c}1.63 \\
(1.14-2.71)\end{array}$ & $\begin{array}{c}1.71 \\
(1.00-2.14)\end{array}$ & 0.5 & 1.57 & 1.79 \\
\hline 10. Irritability Trait & $\begin{array}{c}2.41 \\
(1.43-3.29)\end{array}$ & $\begin{array}{c}2.55 \\
(1.17-3.71)\end{array}$ & $\begin{array}{c}2.86 \\
(1.43-3.57)\end{array}$ & 0.5 & 2.57 & 2.35 \\
\hline 11. Mistrust & $\begin{array}{c}1.60 \\
(1.00-3.14)\end{array}$ & $\begin{array}{c}1.79 \\
(1.00-3.57)\end{array}$ & $\begin{array}{c}1.71 \\
(1.0-3.71)\end{array}$ & 0.3 & 1.71 & 1.93 \\
\hline $\begin{array}{l}\text { 12. Verbal Aggression } \\
\text { Trait }\end{array}$ & $\begin{array}{c}1.95 \\
(1.00-3.00)\end{array}$ & $\begin{array}{c}2.11 \\
(1.00-3.29)\end{array}$ & $\begin{array}{c}2.14 \\
(1.57-3.43)\end{array}$ & 0.3 & 2.14 & 2.10 \\
\hline $\begin{array}{l}\text { 13. Psychological } \\
\text { Aggression Trait }\end{array}$ & $\begin{array}{c}1.54 \\
(1.00-2.29)\end{array}$ & $\begin{array}{c}1.65 \\
(1.00-2.71)\end{array}$ & $\begin{array}{c}1.57 \\
(1.00-3.29)\end{array}$ & 0.4 & 1.43 & 1.89 \\
\hline
\end{tabular}

${ }^{*} \mathrm{P}$ values $<0.05$ are statistically significant.

also with the duration of augmentation. This result is consistent with the impact found in other studies that show that oxytocin affects personal characteristics such as anxiety and the ability to engage in social interaction [2]. We also detected that oxytocin given for a short period at the end of labor does not affect the woman's psychological response to the same degree as more excessive use. The high impact reported was only seen after a prolonged time of augmentation $(\geq 5 \mathrm{~h})$ or for a shorter time but with a higher dose $(40 \mathrm{ml} / \mathrm{h} ; 200 \mathrm{mIU} / \mathrm{h}$ for at least one h).

These data are in line with previous studies showing that the scores of the personality trait inhibition of aggression, obtained by the KSP (Karolinska scales of personality), two days after birth significantly and positively correlated with the dose of oxytocin infused to the women when in labor two days earlier [11].

It is well known that plasma levels of oxytocin rise during pregnancy and reach a maximum (a 3 to 4 -fold rise) at term. The increase in estrogen levels 
causes an increase in oxytocin levels. The amount of oxytocin receptors in the uterine tissue also increases dramatically and reaches its maximum at the end of pregnancy due to high estrogen levels. During labor, oxytocin is released in short spurts with increasing frequency to reach a maximum of 3 pulses $/ 10$ minutes towards the end of the second stage of labor. Just before and during birth, oxytocin levels increase 3 - 4-fold. Oxytocin levels during birth are in part regulated by the Fergusson reflex; afferent sensory nerves are activated when the baby's head is pushing against the cervix and the vaginal wall. Consequently, oxytocin is released [7].

The stronger and more frequent the uterine contractions are, the feed-forward process releases more oxytocin. The base level of oxytocin decreases postpartum, but then oxytocin is released in a pulsatile fashion several times a day in response to breastfeeding.

In work published by Uvnäs-Moberg and Petterson [12], the authors described that, in rats, in addition to the previously known hormonal effects on uterine contraction and milk expulsion, oxytocin causes a variety of behavioral and physiological effects mediated by receptors in the CNS. This effect is probably linked to the brain's amygdala region, the central nucleus rich in oxytocin receptors. The amygdala is believed to have an essential function in the brain's emotional network and plays a significant role in the emergence of negative and positive emotions. The question is whether women receiving oxytocin in higher doses for a more extended period during labor are affected in the same way as the rats described by Uvnäs-Moberg. One can conclude that women who received oxytocin for a more extended period during delivery scored a higher level of "social desirability" and a lower level of anxiety than women who did not receive oxytocin or the control group.

The question is whether this new result should be considered as a positive or a negative outcome. Do we think it is crucial if women are affected by a long augmentation with oxytocin during labor? Less anxiety and more "social desirability" will perhaps be considered an advantage and may assist the woman in her new role as a mother, which also occurs naturally. If this is important, for how long time will the effect remain? Even though previous studies examining oxytocin's psychophysiological impact, very little is known about the actual maternal effects of oxytocin given during labor. Studies show that exogenous oxytocin given during childbirth has a proven correlation to increased oxytocin levels in maternal plasma two months postpartum [2]. In other studies, on the contrary, a negative correlation has been shown between observed intrapartal administered oxytocin and oxytocin levels measured postpartum [3]. The question is whether this is a positive feedback mechanism on endogenous oxytocin secretion from the CNS or an adaptation to higher "base levels" as compared to an acute response with negative feedback. However, it is still unclear how important this will be in a longer perspective.

It has been shown in previous studies that newly delivered women exhibited a different personality profile with less anxiety as well as more social interaction 
compared to a normative group of women [3]. The differences were even more apparent when women delivered by cesarean section were compared with women who were delivered normally. Vaginally delivered mothers showed lower scores in suspicion, anxiety, muscle tension, and higher socialization scores than those delivered operatively. In these studies, the change in personality obtained by the KSP (Karolinska Scale of Personality) was associated with the release of oxytocin occurring during breastfeeding, suggesting that endogenous oxytocin released within the brain in response to suckling was involved in these changes. The interpretation drawn in that study was that the vaginally delivered women were exposed to a more significant oxytocin effect during delivery. Our new findings, that the time and amount of augmentation with exogenous, synthetic oxytocin during labor is essential, maybe another puzzle.

Our results showed that women with an extended augmentation with oxytocin scored lower in terms of anxiety and higher in social conformity, but that the time and amount of augmentation were important. This personality pattern with low anxiety, low suspicion, less guilt, and a high degree of socialization-is commonly regarded as representing mental well-being and is considered an essential part of maternity. The question is, of course, whether the amount of exogenously administered oxytocin and the time of augmentation are of importance.

\section{The Strength of the Study}

Is that the material and the idea are unique and of the highest importance. No similar studies on this issue have previously been performed in humans. The material was collected and unselected consecutively. Swedish women included in the "Dysfunctional Labour Study" received the SSP survey during a limited study period. None of the women had been selected in advance.

\section{The Limitation of the Study}

On the other hand, is the limited number of women that completed the questionnaire and were included in the study. This should be considered when interpreting the results. Further, women included in the study completed the survey 1 - 3 days after childbirth. It would have been desirable to have a corresponding follow-up later in the puerperium. As this follow-up did not happen, the duration of the described personality profile changes among oxytocin-stimulated women during labor is not available. It is still unknown whether the effect is short-term and transient, or permanent. More extensive follow-up studies will, therefore, be performed on this subject.

An important question is also whether it is the situation itself that affects the woman or whether it is the oxytocin. We are not going to get a real answer to this, but this can be seen as a limitation.

This study shows that oxytocin, given as an intravenous infusion during childbirth, affects the woman in anxiety and social conformance. Whether this is a 
positive or an adverse finding is still unknown and warrants further investigation.

We believe that this must be considered an essential question. A large proportion of deliveries are stimulated with synthetic oxytocin during labor, sometimes as many as $70 \%-80 \%$ of all women giving birth. No other obstetric drugs are presented in such large volumes and mainly without the woman's active consent. Studies have also shown that oxytocin is sometimes given routinely and sometimes even unnecessarily. However, in certain situations such as postpartum hemorrhage (PPH), the drug may be a very important maternal lifesaver. Our result is also in line with the Cochran review from 2013 [1]. More vaginal deliveries will not occur, even with this sometimes-excessive use of oxytocin. We hope to gain more knowledge and initiate a critical discussion about how oxytocin should be used most effectively during childbirth. This is important in obtaining a better understanding of this very potent drug and its most secure use.

\section{Conclusion}

This study shows that the volume of oxytocin given and the total time of augmentation during labor are essential when discussing maternal psychological response postpartum. We conclude that prolonged and extended use of synthetic oxytocin during labor should be avoided if possible. We hope that similar studies will be conducted in other clinics and in different settings to confirm our slightly controversial result.

\section{Acknowledgements}

We wish to thank the midwives and nursing staff in the clinic for all their invaluable effort and work. We would also like to thank Marilyn Hedges for language editing.

\section{Conflicts of Interest}

The authors declare no conflicts of interest regarding the publication of this paper.

\section{References}

[1] Bugg, G.J., Siddiqui, F. and Thornton, J.G. (2013) Oxytocin versus No Treatment or Delayed Treatment for Slow Progress in the First Stage of Spontaneous Labor. Cochrane Database of Systematic Reviews, 2013, CD007123. https://doi.org/10.1002/14651858.CD007123.pub3

[2] Uvnäs-Moberg, K., Arn, I. and Magnusson, D. (2005) The Psychobiology of Emotion: The Role of the Oxytocinergic System. International Journal of Behavioral Medicine, 12, 59-65. https://doi.org/10.1207/s15327558ijbm1202_3

[3] Uvnäs-Moberg, K., Widström, A.-M., Nissen, E. and Björvell, H. (1990) Personality Traits in Women Four Days Postpartum and Their Correlation with Plasma Levels of Oxytocin and Prolactin. Journal of Psychosomatic Obstetrics \& Gynecology, 11, 261-173. https://doi.org/10.3109/01674829009084422 
[4] Gu, V., Feeley, N., Gold, I., et al. (2016) Intrapartum Synthetic Oxytocin, and Its Effect on Maternal Well-Being at Two Months Postpartum. Birth, 43, 28-35. https://doi.org/10.1111/birt.12198

[5] Churchland, P. and Winkielman, P. (2012) Modulating Social Behavior with Oxytocin: How Does It Work? What Does It Mean? Hormones and Behavior, 61, 392-399. https://doi.org/10.1016/j.yhbeh.2011.12.003

[6] Pedersen, C.A., Chang, S. and Williams, C. (2014) Evolutionary Perspectives on the Role of Oxytocin in Human Social Behavior, Social Cognition, and Psychopathology. Brain Research, 1580, 1-7. https://doi.org/10.1016/j.brainres.2014.07.033

[7] Ferguson, J.K.W. (1941) A Study of the Motility of the Intact Uterus at Term. Surgery Gynecology, and Obstetrics, 73, 359-366.

[8] Wiberg-Itzel, E., Pembe, A.B., Järnbert-Pettersson, H., et al. (2016) Lactate in Amniotic Fluid: Predictor of Labor Outcome in Oxytocin-Augmented Primiparas' Deliveries. PLoS One, 11, e0161546. https://doi.org/10.1371/journal.pone.0161546

[9] Gustavsson, P., Edman, G., Ekselius, L., et al. (2000) Swedish Universities Scales of Personality (SSP): Construction, Internal Consistency, and Normative Data. Acta Psychiatrica Scandinavica, 102, 217-225. https://doi.org/10.1034/j.1600-0447.2000.102003217.x

[10] Von Elm, E., Altman, D.G., Egger, M., Pocock, S.J., Gotzsche, P.C. and Vandenbroucke, J.P. (2007) The Strengthening the Reporting of Observational Studies in Epidemiology (STROBE) Statement: Guidelines for Reporting Observational Studies. BMJ, 335, 806. https://doi.org/10.1136/bmj.39335.541782.AD

[11] Jonas, W., Nissen, E., Ransjö-Arvidson, A.B., Matthiesen, A.S. and Uvnäs-Moberg, K. (2008) Influence of Oxytocin or Epidural Analgesia on Personality Profile in Breastfeeding. Archives of Women's Mental Health, 11, 335-345, PMID: 18726143. https://doi.org/10.1007/s00737-008-0027-4

[12] Uvnäs-Moberg, K. and Petersson, M. (2004) Oxytocin-Biochemical Link for Human Relations. Mediator of Antistress, Well-Being, Social Interaction, Growth, and Healing. Läkartidningen, 101, 2634-2639. 